\title{
Wavelet analysis on upwelling index along the Moroccan Atlantic coast
}

\author{
Bessa Ismail, M.S \\ Laboratory of engineering and Materials, Hassan II University of \\ Casablanca, Faculty of Sciences Ben M'Sik, P.B 7955, Sidi Othmane, \\ Casablanca, Morocco \\ Makaoui Ahmed, PhD \\ Oceanography Department, National Institute of Fishery Research (INRH), \\ Casablanca, Morocco.

\section{Hilmi Karim, PhD} \\ Afifi Mohamed, PhD \\ Laboratory of engineering and Materials, Hassan II University of \\ Casablanca, Faculty of Sciences Ben M’Sik, P.B 7955, Sidi Othmane, \\ Casablanca, Morocco
}

doi: 10.19044/esj.2017.v13n12p276 URL:http://dx.doi.org/10.19044/esj.2017.v13n12p276

\begin{abstract}
Wavelet analysis is a relatively new technique, an enormous interest in the application of wavelets has been observed in the recent years. In this paper, we use the wavelet analysis on the upwelling index based on sea surface temperature data from 1993 to 2014 along the Moroccan Atlantic coast, mainly at Cape Cantin, Cape Ghir, Cape Juby, Dakhla and Cape Blanc. We noticed that from Jan 1993 to Oct-1998 and from Sep-2007 to Nov 20014, the upwelling in the five research latitudes increases with fluctuations. The period of Upwelling varies sensitively with the change of time scale. The value of upwelling index in the five latitudes appears similar and present an obvious period of one year in all latitudes studied. This study can be considered an important tool for time series analysis, which can help the studies concerning the upwelling variability.
\end{abstract}

Keywords: Sea Surface Temperature, Upwelling index, Wavelet analysis, Upwelling.

\section{Introduction}

Wavelet analysis is a powerful technique for signal processing that was extensively developed only as recently in the 1980s. Its uniqueness is its capability of simultaneously localizing the variability of the signal in both 
time and frequency domains (Mak 1995). They can reveal the frequency characteristic at any times, with higher resolution (Jintai 1995). Furthermore, they can analyze the signal in detail at many scales. For the reason of the multiple solution and adaptive, wavelet analysis plays an important part in time series analysis, predicting the period or trend (Chang et Pedro 1997).

The Moroccan coast is one of the major coastal upwelling regions of the world (Thomas, et al. 2014) (Makaoui, et al. 2012) (Makaouia, et al. 2005). The upwelled water is typically denser, cooler and richer in nutrients than surface waters and has significant impacts on coastal climates and marine biology (Miranda, Alves et Serra 2012). Moreover, the upwelling activity influence the variability of the ocean mixed layer depth (Bessa, et al. 2017).

Consequently, the activity of the upwelling is an important socioeconomic, oceanographic and climatologically region.

The upwelling ecosystems cover approximately $1 \%$ of the total ocean surface but account for over $20 \%$ of the global fish catch (Pauly, and Christensen, 1995). Therefore, monitoring changes across these regions is of the upmost importance, especially as fisheries response to global climate change has recently been documented (Payne 2013)_(Cheung, et al. 2013).

In this paper, we use Mexican hat function (Mexhat) to investigate the changes in the upwelling index based on the SST satellite data, in Cape Cantin, Cape Ghir, Cape Juby, Dakhla and Cape Blanc located in the Moroccan Atlantic coast region. In addition, the figures of wavelet variance shows the probable period. Finally, we compared the variance of trend among the five located areas.

\section{Data and method}

With the data from Armor-3D L4 Combined products from satellite observations Sea Surface Temperature and in-situ (Temperature ${ }^{\circ} \mathrm{C}$ and Salinity (PSU) profiles). On a $1 / 4$ degree regular grid over the time period 1993-2014. We used the monthly average data (Guinehut, et al. 2012).

\subsection{The upwelling SST index}

The SST index of upwelling at each latitudinal point is defined as the difference in SST between the coast and the ocean (Rayner, et al. 2003) (Reynolds, et al. 2007)

$$
\text { UIsst }=\text { SSTocean }- \text { SSTcoast }
$$

Where SSTcoast is the SST of the grid closest to the coast and SSTocean is the SST of the grid box along the same latitude that is $5^{\circ}$ to the west. Therefore, an increase (decrease) in the UIsst is equivalent to a decrease (increase) in the intensity of the upwelling. 


\subsection{A Brief Introduction about wavelet}

If the $\Psi$ is an energy limited function, which is defined in $(-\infty,+\infty)$, composing square integral signal space $(\Psi \in \mathrm{L} 2(\mathrm{R}))$, then the function group can be created:

$$
W T(f(t) ; a, b)=\int_{R} f(t) \Psi\left(\frac{t-b}{a}\right) d t
$$

In this function, $\Psi(\mathrm{t})$ can be called wavelet function. After the flex and translation, we get $\Psi_{\mathrm{a}, \mathrm{b}}(\mathrm{t})$, which is called wavelet base function, $a$ is the flex function, $b$ is translation factor (CX. 2001).

Wavelet Transform is a linear decomposition operations on function $\mathrm{f}(\mathrm{t}) \in \mathrm{L}_{2}(\mathrm{R})$, using wavelet function group $\Psi_{\mathrm{a}, \mathrm{b}}(\mathrm{t})$ in different scales. In addition, it can be defined as the inner product of the signal and wavelet base function:

$$
W T(f(t) ; a, b)=|a|^{-1 / 2} \int_{R} f(t) \Psi\left(\frac{t-b}{a}\right) d t
$$

The wavelet variance reveals the energy distribution in different time scales, reflecting the relative intensity of the disturbance at a time series. Form that, we can used in Continuous transformation; the generating function is (Yuanping et Taibao 2007).

$$
\varphi(t)=\left(1-t^{2}\right) e^{\frac{-t^{2}}{2}}
$$

\subsection{Research Area}

The five areas are located at $32.25^{\circ} \mathrm{N}, 31^{\circ} \mathrm{N}, 28.25^{\circ} \mathrm{N}, 25.25^{\circ} \mathrm{N}$ and $21^{\circ} \mathrm{N}$. Those latitudes were selected in the areas of the intense upwelling activity described by (Makaouia, et al. 2005).

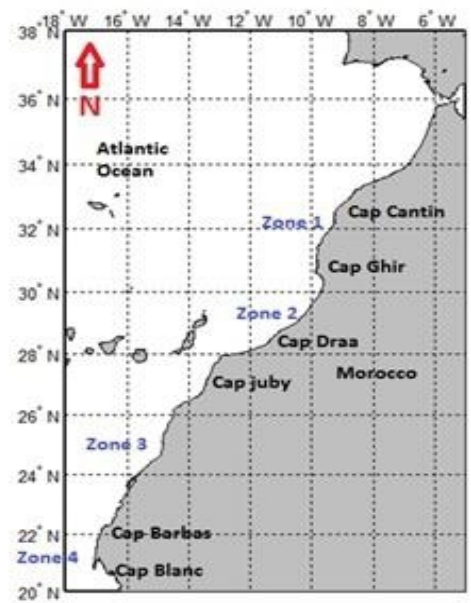

Figure 8 : The research areas and the zones of the upwelling located between capes 


\section{Results and discussion}

2.1. Characteristics variation of the Upwelling index in the $32.25^{\circ} \mathrm{N}$ Fig. 2 shows the variation of the upwelling index from Jan. 1993 to Nov. 2014 in the Cape Cantin Area. At this period, the UIsst show a significant seasonality of the Upwelling. The minimum values of the UIsst appear in winter season and the maximum values appear at summer season. The lowest value of the UIsst is remarked in May 1994 and Mars 2000, with a value of $0^{\circ} \mathrm{C}$ and $0.1^{\circ} \mathrm{C}$. On the other hand, highest value was noticed in Aug 2004 and Aug 2012 , with $3.7^{\circ} \mathrm{C}$ and $3.5^{\circ} \mathrm{C}$ respectively. In general, the UIsst trend is regular. The UIsst is higher in summer and lower in winter.

Fig. 3 (Above) is the results of the wavelet Transform. In this figure, the yellow part refers to the area that the column density is higher than the average value, in contrast, the red part refers to the column density is lower than the average value. In short time-scale, the oscillating period of the wavelet is obvious. In long time-scale, the oscillating period is obscure because of containing complex variations in short time scale. At the time scales of 32 months, the value before Sept 1999 is on the high side. From Sept 1999 to Jan 2000, the value is on the low side. From Jan 2000, to Feb 2006, it becomes higher. From Feb 2006 to Avril 2009, it becomes lower and from Nov 2008, to Nov 2014 it become higher again. In addition, at the time scale of 6 months, the period is also obvious, with a period about one year. In Short, it present approximately a 3 years and one-year period.

Fig 3 (below) shows the wavelet variance of UIsst. The time series contains tree wave crests, corresponding to the period of 37 months, 137 months and 240 months. Between the 3 periods, the latter is the main period. In addition, on large time scale, UIsst may increase slowly in several years.

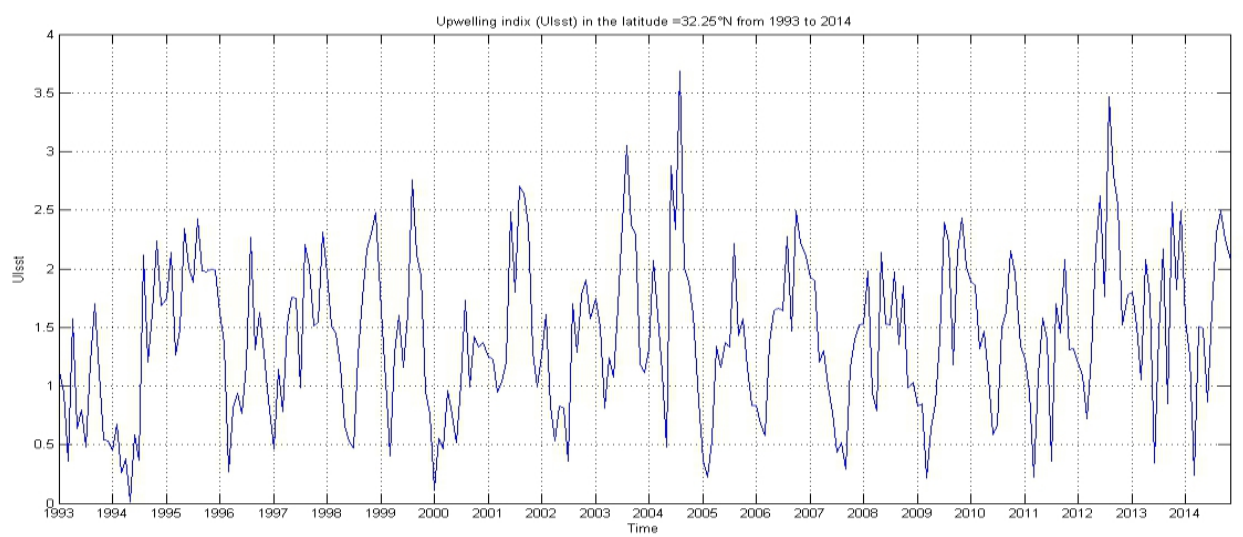

Figure 9 : Time series of UIsst in the latitude $32.25^{\circ} \mathrm{N}$ 
Ca,b Coefficients - Coloration mode: init + by scale + abs
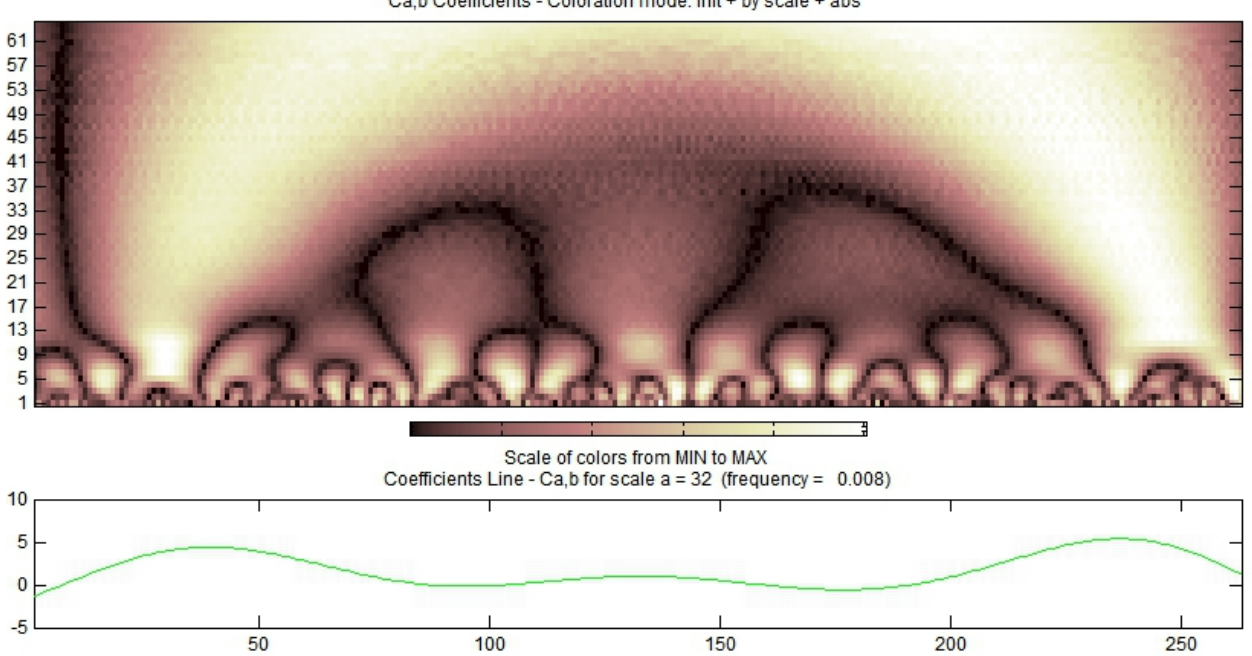

Figure 10 : Wavelet transform coefficient (above) and the wavelete variance (below) of the UIsst in the $32.25^{\circ} \mathrm{N}$

\subsection{Variation characteristics of the Upwelling index in the $31 \mathrm{~N}^{\circ}$}

Using the same method, we take the wavelet transform on the UIsst in the Cape Ghir region from Jan 1993 to Nov 2014. Fig 4 shows the variation trend. The fluctuation trend is obvious and regular, with high variable amplitude than that in the Cape Cantin. The highest value usually appears at Jun, Jull or Aug, and the lowest value appears at Jan, Feb and Mar. the maximum value of the UIsst reaches to $5.88^{\circ} \mathrm{C}$ and $5.3^{\circ} \mathrm{C}$ in Aug 2004 and Nov 2013, respectively and the minimum in Feb 1994 close to $0.3^{\circ} \mathrm{C}$.

The results of the wavelet transform on UIsst are listed in Fig 5. The wavelet transform coefficient of UIsst in the Cape Ghir region is similar to that in Cape Cantin. In long time scale, the UIsst varies frequently so the period is not obvious. In the other hand, we notice two periods at the time scales of 29 months and 40 months respectively. From Jan 1993 to Aug 1999 the UIsst value is on the high side, followed by a lower period from Aug 1999 to Jull 2001, and from Jul 2001 to Jan2006the UIsst value is high, followed by a low period from Jan 2006 to May 2008, the value of the UIsst increase from May 2008 to Nov 20014. On small time-scale, the period is obvious, with a period about one year.

In addition, we get variance of the above-region value. In Fig 5 (below), we find tree wave crests, reflecting tree periods: 37 months, 133 months and 244 months, and the 244 months is the main period. In 3 years scale, the Upwelling will decrease. In 11 years, the Upwelling will undergo a small increase in 133 months and in 244 months; the upwelling will increase after a period of reduction. 


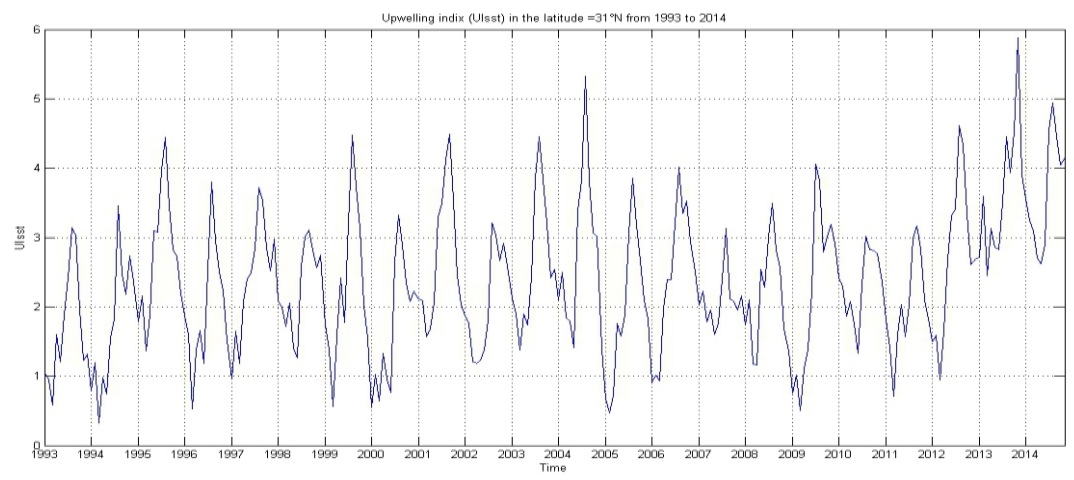

Figure 11 : Time series of UIsst in the latitude $31^{\circ} \mathrm{N}$
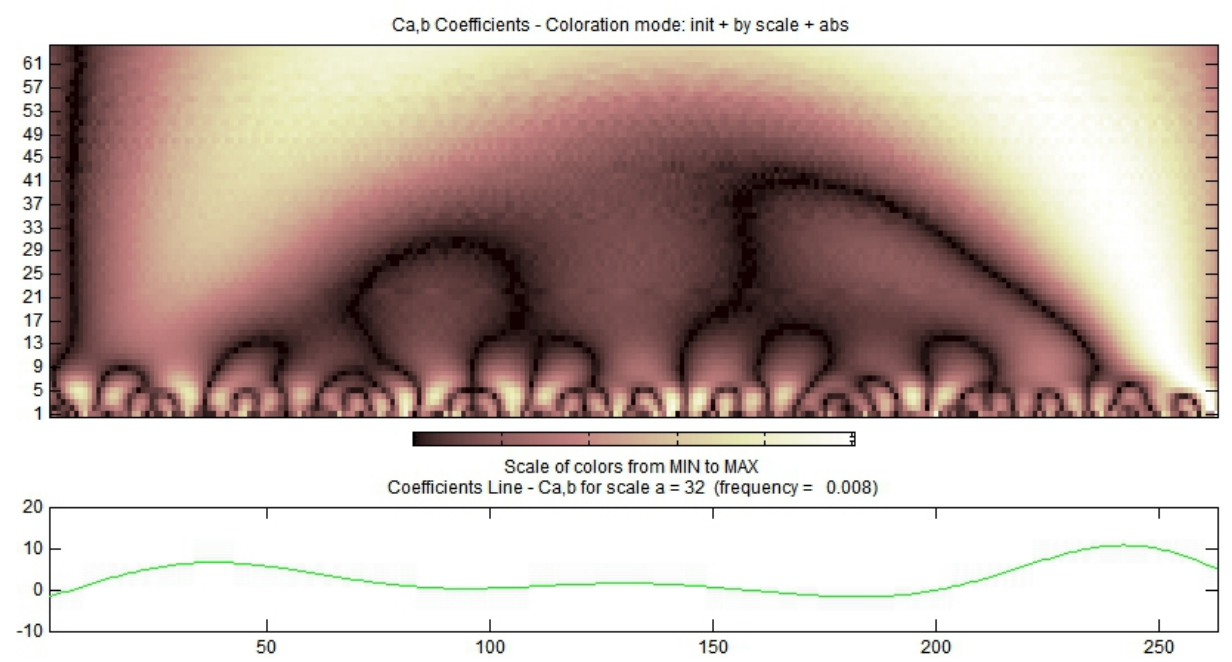

Figure 12 : Wavelet transform coefficient (above) and the wavelete variance (below) of the UIsst in the $31^{\circ} \mathrm{N}$

2.3. Variation characteristics of the Upwelling index in the $28.25 \mathrm{~N}^{\circ}$

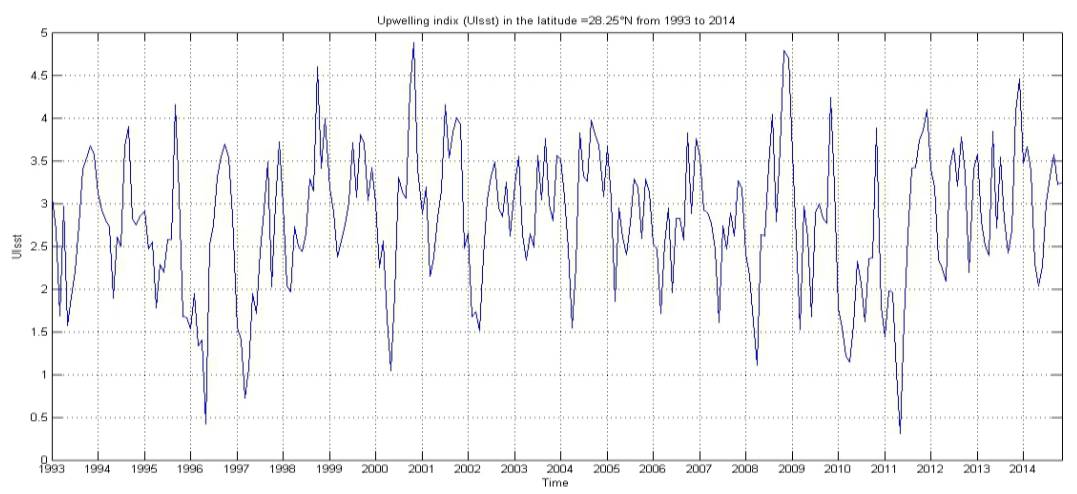

Figure 13 : Time series of UIsst in the latitude $28.25^{\circ} \mathrm{N}$ 
Ca,b Coefficients - Coloration mode: init + by scale + abs
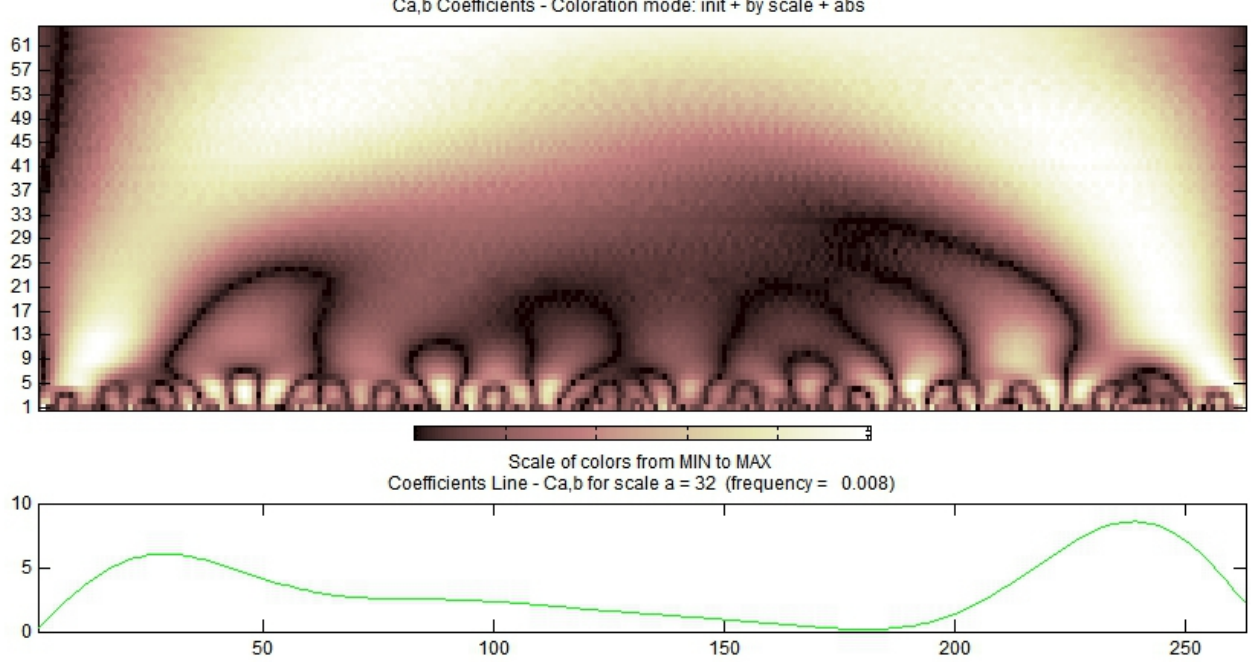

Figure 14 : Wavelet transform coefficient (above) and the wavelete variance (below) of the UIsst in the $28.25^{\circ} \mathrm{N}$

Using the same method, we do the same wavelet transform on the Upwelling index in the Cape Juby $28.25^{\circ} \mathrm{N}$ from Jan 1993 to Nov 2014. Fig 6 shows the variation trend of the UIsst. The fluctuation trend is not regular in this region, with the highest value appears at Aug and Nov., and the lowest value appears at Feb. Apr and May, in Apr 1996 and May 2011 the value of the UIsst reaches $0.4^{\circ} \mathrm{C}$ and $0.3^{\circ} \mathrm{C}$, respectively. In contrast, on Nov 2000 and Dec-2008 the value reaches the $4.8^{\circ} \mathrm{Cand} 4.7^{\circ} \mathrm{C}$, respectively.

The results of the wavelet transform on UIsst is listed in Fig. 7 (above). In long time scale, wavelet transform coefficient of UIsst in this region is not similar to that in the Cape Ghir. In long time scale, the UIsst varies frequently so the period is not obvious. We can notice many periods for different scales from 19 months to 32 months but in the same time in the scale of 6 months, the period in clear about one year.

Fig 7 (below) shows the wavelet variance of the Upwelling index. The time series contains two waves crests, corresponding to period of 27 months and 239 months. The main period is 239 months, the upwelling increase after a long decrease.

\subsection{Variation characteristics of the Upwelling index in the $25.25 \mathrm{~N}^{\circ}$} Using the same method, we do the same wavelet transform on the Upwelling index in the Dakhla $28.25^{\circ} \mathrm{N}$ from Jan 1993 to Nov 2014. Fig 8 shows the variation trend of the UIsst. The fluctuation trend is regular in this region, with the highest value appears at Jun and Aug., and the lowest value appears at Jan to Apr., in Apr-1993 and Mar-1994the value of the UIsst reaches to 
$0.2^{\circ} \mathrm{C}$ and $0.3^{\circ} \mathrm{C}$, respectively. In contrast, on Jun-2012 and Aug-2014 the value reaches the $5.2^{\circ} \mathrm{C}$ and $5^{\circ} \mathrm{C}$, respectively.

The results of the wavelet transform on UIsst is listed in Fig 9 (above). In long time scale, wavelet transform coefficient of UIsst in this region is similar to that in the Cape Ghir. In long time scale, the UIsst varies frequently so the period is not obvious. We can notice two periods for different scales of 37 months to 45 months. At the scale of 10 months, we have a regular period, but the period is clear in the scales of about one year.

Fig 9 (below) shows the wavelet variance of the Upwelling index. The time series contains tree wave's crests, corresponding to period of 40 months, 137 months and 239 months. The main period is 239 months, the upwelling the Upwelling will undergo a small increase 137 and in 244 months, the upwelling will increase after a period of decrease.

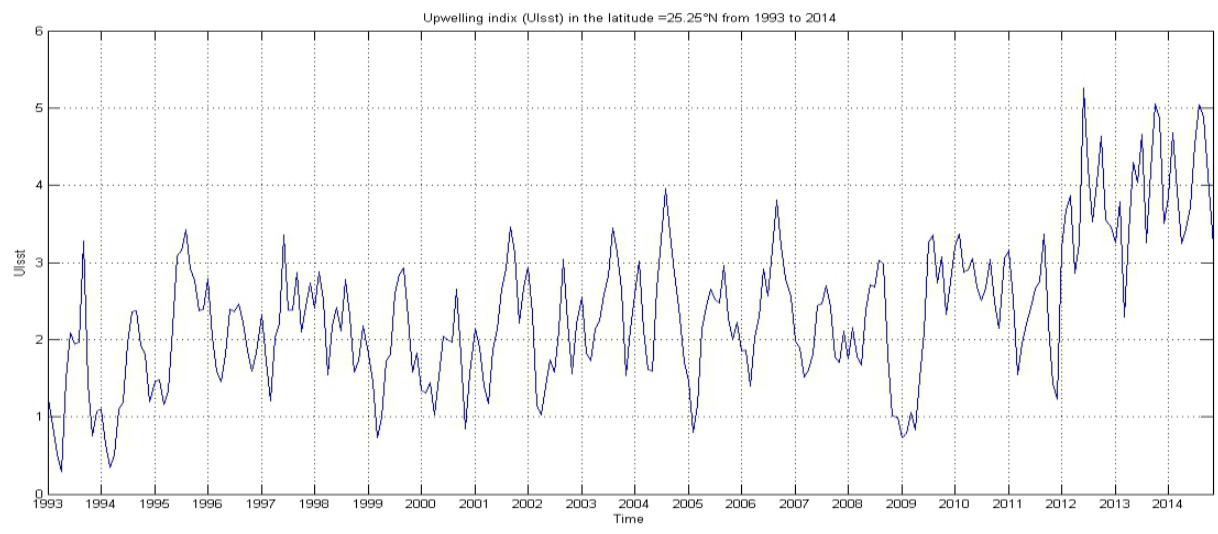

Figure 15 : Time series of UIsst in the latitude $25.25^{\circ} \mathrm{N}$

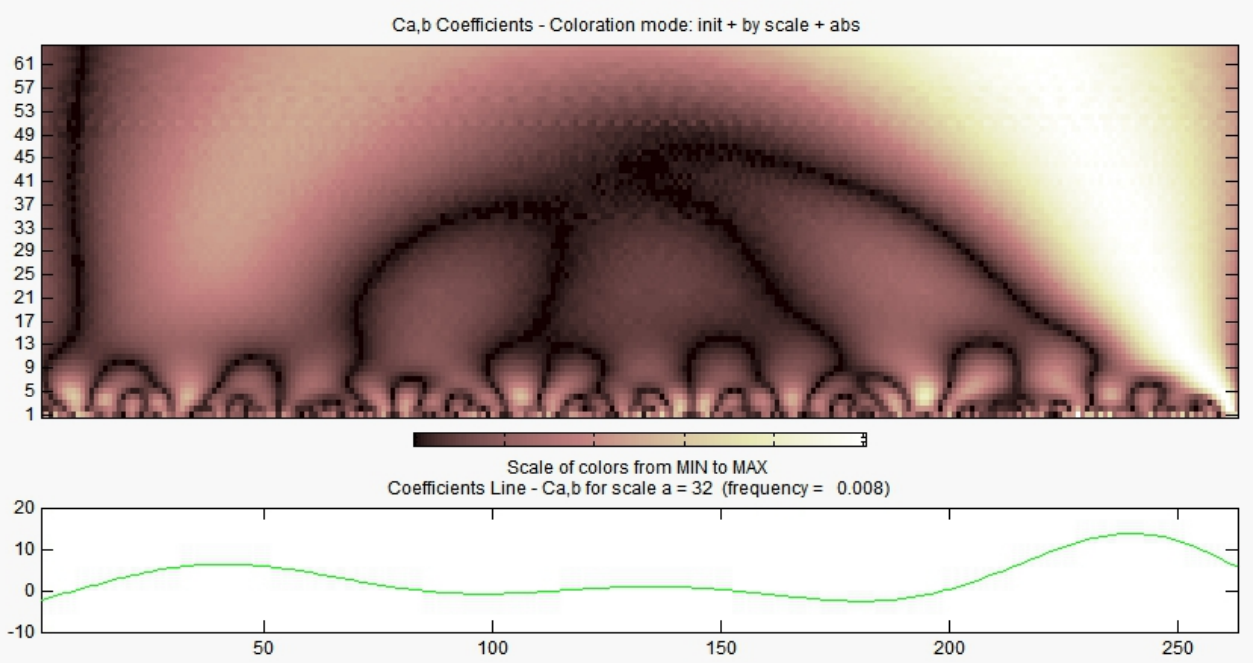

Figure 16: Wavelet transform coefficient (above) and the wavelete variance (below) of the UIsst in the $25.25^{\circ} \mathrm{N}$ 
2.5. Variation characteristics of the Upwelling index in the $21 \mathrm{~N}^{\circ}$

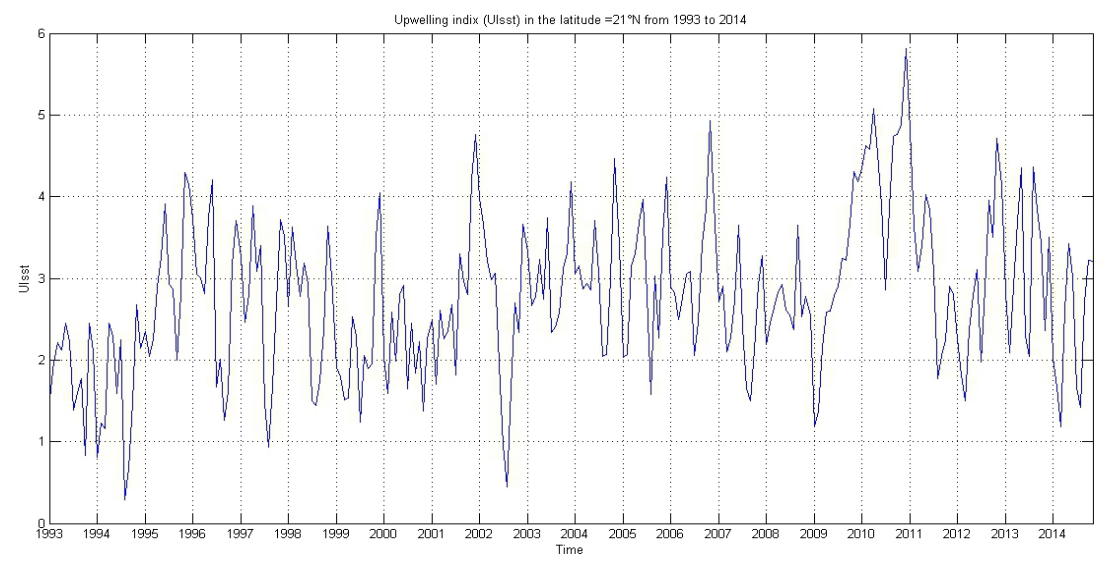

Figure 17 : Time series of UIsst in the latitude $21^{\circ} \mathrm{N}$

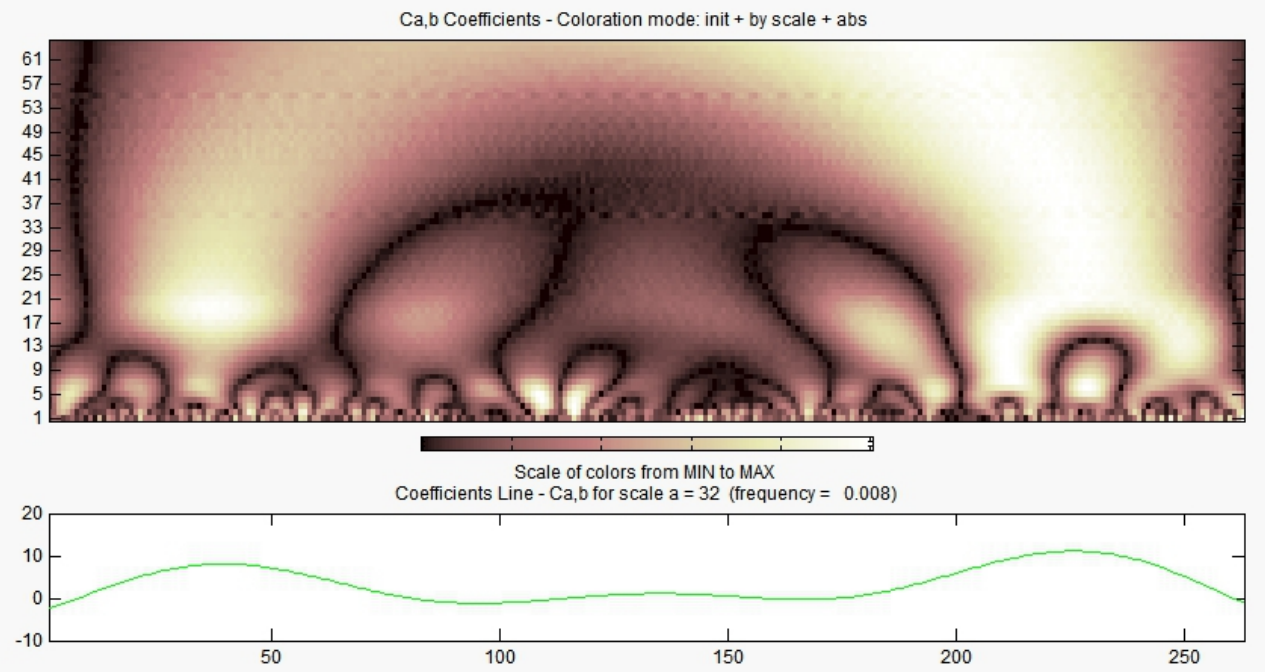

Figure 18 : Wavelet transform coefficient (above) and the wavelete variance (below) of the UIsst in the $21^{\circ} \mathrm{N}$

Using the same method, we take the wavelet transform on the UIsst in the Cape Blanc region from Jan 1993 to Nov 2014. Fig 10 shows the variation trend. The fluctuation trend is obvious and regular, with a high variable amplitude than that in the Cape Cantin. The highest value usually appears at Jun, Jull or Aug, and the lowest value appears at Jan, Feb and Mar. the maximum value of the UIsst reaches to $5.88^{\circ} \mathrm{C}$ in Dec-2010, and the minimum in Aug-1994 close to $0.2^{\circ} \mathrm{C}$.

The results of the wavelet transform on UIsst is listed in Fig 11(above). The wavelet transform coefficient of UIsst in the Cape Blanc region is similar to that is Dakhla. In long time scale, the UIsst varies 
frequently so the period is not obvious. In the other hand, we notice two periods at the time scales of 38 months and 33 month. From Jan 1993 to Oct-1998the UIsst value is on the high side, followed by a lower period from Oct-1998to May-2002, and from May-2002 to Nov-2005 the UIsst value is high, followed by a low period from Nov-2005 to Sep-2007, the value of the UIsst increase from Sep-2007 to Nov 20014. On small time-scale, the period is obvious, with a period about 6 months.

In addition, we get variance of the above-region value. In Fig 11 (below), we find tree wave crests, reflecting tree periods: 38 months, 134 months and 227 months, and the 227 months is the main period. In 3 years scale, the Upwelling will decrease. In 134 months, the Upwelling will go through a small increase and in 227 months, the upwelling will increase after a small period of reduction.

2.6. Comparison of the upwelling index among the five studied latitudes Fig.12 shows the variation of upwelling index in the five research latitudes from Jan 1993 to Nov 2014. The value of the UIsst in the latitudes 21, 25.25, 28.25 and $31^{\circ} \mathrm{N}$ have the same order of magnitude, while the value of the UIsst in the $32.25^{\circ} \mathrm{N}$ is considerably smaller. The low value is located at the Cape Cantin region. We can notice the regularity of the upwelling activity in the five researches area the lower value of the UIsst appears in Jan, Fev, and Mar and the higher value appears in Jun, Jul, and Aug. The difference between activities of the upwelling in the regions is especially in the amplitude and in general, the upwelling has a period of one year.

Figure 19 : Comparison on the UIsst variability among the five latitudes

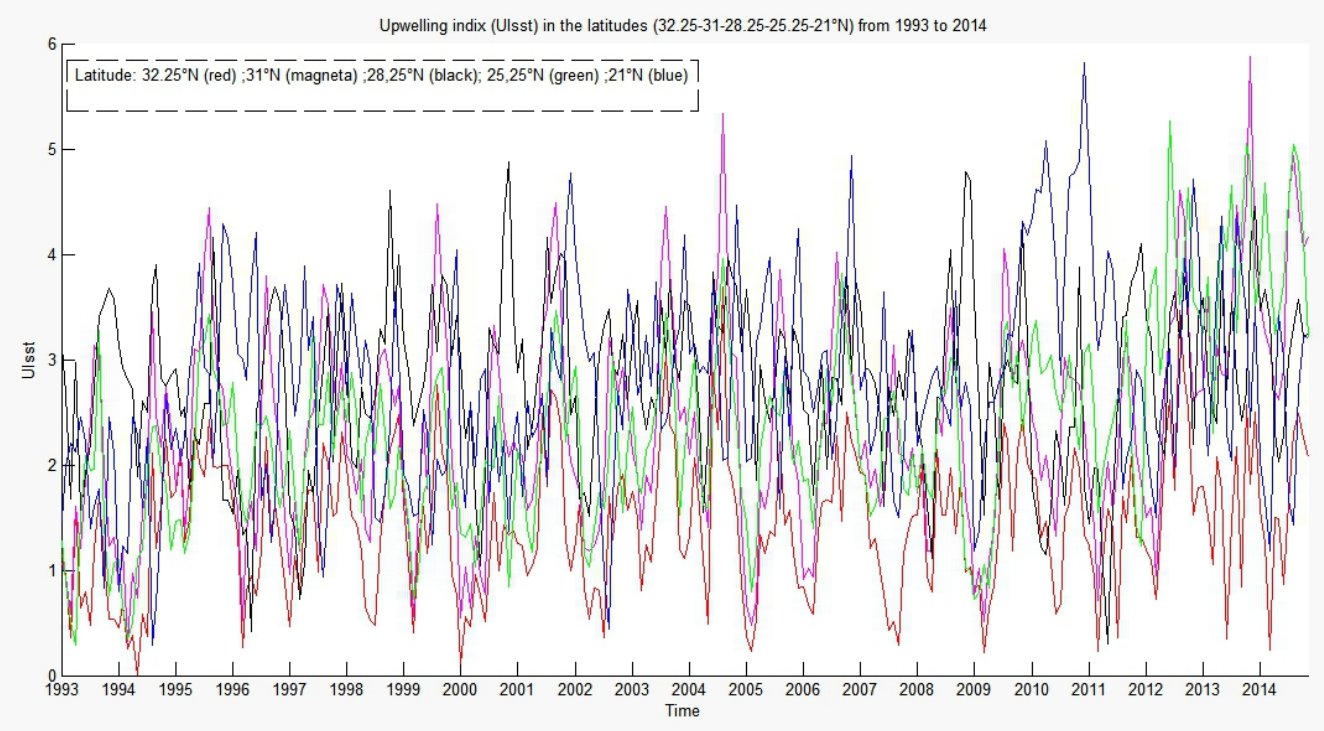

4. Conclusion 
We used the wavelet analysis on the upwelling index in different latitudes from $32.25^{\circ} \mathrm{N}$ to $21^{\circ} \mathrm{N}$ in the Moroccan Atlantic coast, using Mexican hat. After that, we get probable periods and variation trend of them. The whole experiment shows that:

a) From Jan 1993 to Oct-1998 and from Sep-2007 to Nov 20014, the upwelling in the five research latitudes increases with fluctuations.

b) The period of Upwelling varies sensitively with the change of time scale. The value of UIsst in the five latitudes appears similar with a possible period of 3 years at the time-scale about 32 months. At small time-scale of 6 months, the value of the UIsst present an obvious period of one year in the all latitudes studied.

c) With respect of the wavelet variance, 4 research areas are all have 3 possible periods locating in time scale about 37 months, 137 months and 240 months in Cape Cantin, 34 months, 133 months and 244 months in Cape Ghir. In the region of Dakhla, we have a 40 months, 137 months and 239 months. In Cape Blanc, we have 38 months, 134 months and 227 months. In the Cape Juby, we noticed that we have two possible periods, in 27 months and 239 months we must notice that this region is near to the Canary Islands. The main period is about 3 years.

d) In three or four years, the upwelling activity in the five research areas may decrease gradually followed by an increase.

In conclusion, we do the preliminary research on period and variation trend of the upwelling index in five latitudes. In addition, we predict the possible change in the future. Variation of the upwelling index may be related to the Canary courant, amount of sand and sediment, rainfall, and salinity, which need more research, combining remote sensing data model of the SST.

\section{Acknowledgements}

- This study has been conducted using E.U. Copernicus Marine Service Information data

\section{References :}

1. Bessa, Ismail, et al. "SEASONAL VARIABILITY OF THE OCEAN MIXED LAYER DEPTH IN MOROCCAN UPWELLING AREAS DERIVED FROM IN SITU PROFILES." Int. J. of Adv. Res, 2017: 5 (3). 1754-1762, DOI: 10.21474/IJAR01/3688.

2. Chang, C, and AM Pedro. "A wavelet analysis for time series." 1997: 15-32.

3. Cheung, W,W,L., R, Watson, and D, Pauly. "Signature of ocean warming in global fisheries catch." Nature, 2013: 497, 365-368. 
4. CX., L. "Wavelet analysis on interannual change of tropical cyclone in Guangdong." Journal of Tropical Meteorology, 2001: 381-90.

5. Guinehut, S, L Dhomps A, G Larnicol, and P,Y, Le Traon. "High resolution $3 \mathrm{D}$ temperature and salinity fields derived from in situ and satellite observations." Ocean Sci, 2012: 845-857.

6. Jintai, C. An Introduction to Wavelets. Xi'an, 1995.

7. Mak, Mankin. "Orthogonal Wavelet analysis : interannual variability in the sea surface temperature." Bulletin of the American Metreorological society, 1995: 2179.

8. Makaoui, Ahmed, et al. "Hydrological seasonality of cape Ghir filament in Morocco." Hydrological seasonality of cape Ghir filament in Morocco. No.1, no. Vol.4, (2012): 5-13.

9. Makaouia, Ahmed, Orbia Abdelattif, Karim Hilmia, Soukaina Zizaha, Jamila Larissia, and Mohammed Talbib. "L'upwelling de la côte atlantique du Maroc entre 1994 et 1998." Comptes Rendus Geoscience Volume 337, Issue 16 (2005): Pages 1518-1524.

10. Miranda, P.M.A., J.M.R. Alves, and N Serra. "Climate change and upwelling: response of Iberian upwelling to atmospheric forcing in a regional climate scenario." Clim. Dyn., 2012: 40, 2813-2824 .

11. Pauly, D., and V., Christensen. "Primary production required to sustain global fisheries." Nature , 1995. : 374, 255-257.

12. Payne, M.R. "Climate change at the dinner table." Nature , 2013: 497, 320-321.

13. Rayner, N.,, et al. "Global analyses of sea surface temperature, sea ice, and night marine air temperature since the late nineteenth century." Geophys., 2003: 4407.

14. Reynolds, R.W.,, T.M., Smith, C., Liu, D.B., Chelton, K.S., Casey, and M.G., Schlax. "Daily high-resolution-blended analyses for sea surface temperature." J. Clim, 2007: 5473-5496.

15. Thomas, E, Cropper n, Edward Hanna, and Grant R. Bigg. "Spatial and temporal seasonal trends in coastal upwelling off Northwest Africa, 1981-2012." Deep-Sea Research I, 2014: 86 94-111.

16. Yuanping, L, and Y Taibao. "Wavelet analysis on temperature and rainfall in Qaidam Basin for 50 years." Arid Land Geography, 2007: 708-13. 